\title{
Excitonic Aharonov-Bohm effect: Unstrained versus strained type-I semiconductor nanorings
}

\author{
M. Tadić, ${ }^{1, *}$ N. Čukarić, ${ }^{1, \dagger}$ V. Arsoski, ${ }^{1, \dagger}$ and F. M. Peeters ${ }^{2, \S}$ \\ ${ }^{1}$ School of Electrical Engineering, University of Belgrade, P.O. Box 35-54, 11120 Belgrade, Serbia \\ ${ }^{2}$ Department of Physics, University of Antwerp, Groenenborgerlaan 171, B-2020 Antwerp, Belgium \\ (Received 10 June 2011; revised manuscript received 3 August 2011; published 9 September 2011)
}

\begin{abstract}
We study how mechanical strain affects the magnetic field dependence of the exciton states in type-I semiconductor nanorings. Strain spatially separates the electron and hole in (In,Ga)As/GaAs nanorings which is beneficial for the occurrence of the excitonic Aharonov-Bohm (AB) effect. In narrow strained (In,Ga)As/GaAs nanorings the $\mathrm{AB}$ oscillations in the exciton ground-state energy are due to anticrossings with the first excited state. No such $\mathrm{AB}$ oscillations are found in unstrained $\mathrm{GaAs} /(\mathrm{Al}, \mathrm{Ga}) \mathrm{As}$ nanorings irrespective of the ring width. Our results are obtained within an exact numerical diagonalization scheme and are shown to be accurately described by a two-level model with off-diagonal coupling $t$. The later transfer integral expresses the Coulomb coupling between states of electron-hole pairs. We also found that the oscillator strength for exciton recombination in (In,Ga)As/GaAs nanorings exhibits AB oscillations, which are superimposed on a linear increase with magnetic field. Our results agree qualitatively with recent experiments on the excitonic Aharonov-Bohm effect in type-I (In,Ga)As/GaAs nanorings.
\end{abstract}

DOI: 10.1103/PhysRevB.84.125307

PACS number(s): 73.21.La, 78.67.Hc

\section{INTRODUCTION}

Fabrication of nanometer-sized semiconductor rings triggered interest in the excitonic Aharonov-Bohm (AB) effect. ${ }^{1-7}$ Simple theoretical models predict oscillations in the exciton energy in one-dimensional (1D) rings when the magnetic field through the ring varies. ${ }^{3,4}$ But further theoretical work showed no oscillations in the exciton ground-state energy of type-I 2D and 3D nanorings, ${ }^{8,9}$ or they were found to be extremely small. ${ }^{5}$ An interesting analytical model of two concentric 1D rings, where the electron and the hole are confined on separate rings, but are Coulomb coupled leading to the formation of an exciton, showed that the emission intensity for recombination of this exciton vanishes in certain ranges of magnetic field, which is called the optical excitonic AB effect. ${ }^{10}$ However, these bright-to-dark transitions are found only for the case of weak effective Coulomb interaction, i.e., when radii of the two rings are small. ${ }^{10}$ Furthermore, this analytical model showed that in order to find the optical excitonic AB effect, one should polarize the exciton by confining the electron and the hole in spatially separate potentials. This condition is very difficult to fulfill in type-I semiconductor nanorings, where the electron and the hole are radially confined in the same space. The quest for the $\mathrm{AB}$ effect for neutral excitons in type-I nanorings was not successful until recently. ${ }^{11,12}$ The electron and hole ground states exhibit orbital momentum transitions, which give rise to oscillations in the exciton energy levels with magnetic field, which is a fingerprint of the $\mathrm{AB}$ effect.

An appealing and elegant way to polarize the exciton is realized in type-II nanodots, which confine the electron (hole) inside the dot, whereas the hole (electron) is expelled to the region outside the dot. ${ }^{13,14}$ Nevertheless, the latter is confined due to the Coulomb interaction with the former. Such confinement establishes favorable conditions for the occurrence of the optical excitonic AB effect. However, no bright-to-dark transitions were found and experiments on different systems showed some contradictory details. As an example, Ref. 14 found that oscillations in the emission intensity of stacks of $\mathrm{ZnTe} / \mathrm{ZnSe}$ nanodots are superimposed on a decaying function with magnetic field. On the other hand, the photoluminescence intensity in a single InP/GaAs quantum dot was found to decrease periodically in narrow ranges of magnetic field, and to increase quasilinearly between these drops. ${ }^{15}$ A similar increasing trend with superimposed oscillations was recently found in an experiment on InGaAs/GaAs nanorings. ${ }^{11}$

In a beautiful experiment on (In, Ga)As/GaAs nanorings, Bayer et al. found $\mathrm{AB}$ oscillations in the ground state of a charged exciton. ${ }^{16}$ However, the ground state of the neutral exciton did not show any oscillations, or they were too small to be detected experimentally. Those rings were fabricated by means of lithography and had width of the order of $30 \mathrm{~nm}$. Much narrower strained (In,Ga)As/GaAs nanorings were fabricated by means of epitaxy in the Stranski-Krastanov mode, ${ }^{1}$ which allows them to self-assemble on lattice mismatched substrates. Recent experiments, using cross-sectional scanning tunneling microscopy (X-STM), found that these strained self-assembled nanorings have volcano-like shapes, with lateral width of about $7 \mathrm{~nm}$. They are formed from self-assembled quantum dots, by removing the material in the dot center. The process is driven by strain, and a thin layer of nonuniform thickness resides in the ring opening. Therefore, these rings are not fully opened and could be understood as combinations of a nanoring with a nanodot inside the ring opening. ${ }^{17}$ The electron and the hole wave functions extend to the region of the nanodot, and the transition of the orbital momenta in the electron ground state shifts toward larger magnetic fields. ${ }^{18,19}$ In addition to the Stranski-Krastanov growth, an interesting new method to create nanorings is by the technique of droplet epitaxy. ${ }^{20}$ These rings are usually based on an unstrained $\mathrm{GaAs} /(\mathrm{Al}, \mathrm{Ga}) \mathrm{As}$ heterostructure and are typically larger than the strained (In,Ga)As/GaAs nanorings.

In this paper, we investigate the influence of strain on the exciton states in type-I nanorings in the presence of a normal magnetic field. We compute the exciton states in (1) an (In,Ga)As nanoring embedded in a GaAs matrix, and (2) a GaAs nanoring embedded in an (Al,Ga)As matrix. The geometry of both rings and their characteristic dimensions are displayed in Fig. 1(a). They are generated by revolving a 
(a)

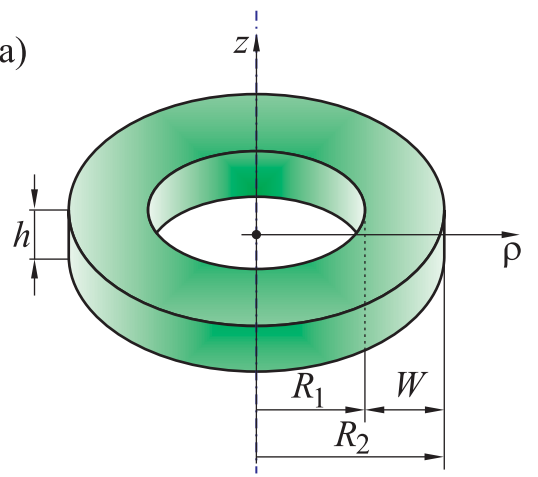

(b)

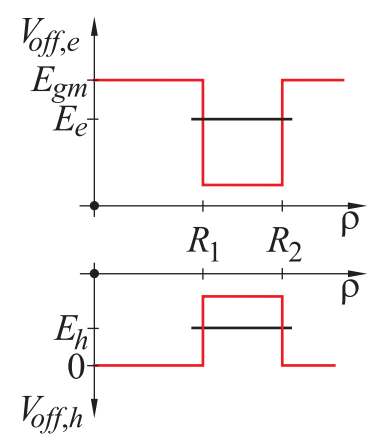

FIG. 1. (Color online) (a) The geometry of the nanoring. (b) The confining potentials due to the band offsets as they vary along the $\rho$ axis $(z=0)$. The electron and hole energy levels, $E_{e}$ and $E_{h}$, are measured with respect to the top of the valence band in the semiconductor matrix.

rectangle of height $h$ and width $W$ about the $z$ axis. The inner radius of the ring is $R_{1}$ and the outer radius is $R_{2}=R_{1}+W$. Energy is measured with respect to the top of the valence band in the matrix material, and the energy axis for holes points downward, as shown in Fig. 1(b). Figure 1(b) also shows the energy level of the electron $E_{e}$, the hole energy $E_{h}$, and the energy of the bottom of the conduction band in the matrix $E_{g m}$. Furthermore, the potentials due to the offsets of the conduction and valence band, $V_{\text {off, } e}$ and $V_{\text {off, } h}$, respectively, as they vary with $\rho$ for $z=0$ are depicted in Fig. 1(b). In the case of the (In,Ga)As nanoring the rectangular potential wells shown in Fig. 1(b) are modified by strain, which arises from the lattice mismatch between (In,Ga)As and GaAs. The strain distribution is extracted from the continuum mechanical model, and the finite element method (FEM) is employed to discretize the components of the displacement vector on a nonuniform mesh. ${ }^{21}$ The $\mathrm{GaAs} /(\mathrm{Al}, \mathrm{Ga}) \mathrm{As}$ nanorings are taken to be strain free and are assumed to have the same dimensions as the (In,Ga)As/GaAs nanorings. The effects of strain are studied as a function of the ring dimensions, and its consequences for the variations of the exciton energy levels with magnetic field are critically examined.

The effective-mass Schrödinger equations for the electron and the hole are solved by FEM on the same mesh used to compute the strain distribution. Products of the electron and hole wave functions form the basis to construct the full exciton Hamiltonian matrix. However, such an exact diagonalization scheme lacks a simple parametrization of the exciton energy levels. Therefore, we employ Löwdin perturbation theory to construct the approximate exciton Hamiltonian which has the form of a tight-binding model, and takes into account the interaction between the two lowest energy states of the electron-hole pair. This model is employed to explain both the observed anticrossings between the exciton energy levels and the role of strain on the exciton $\mathrm{AB}$ oscillations. Furthermore, from the exciton wave function, the oscillator strength for exciton recombination is calculated. The exciton energy levels and the oscillator strength are determined as they vary with magnetic field, for a range of 3D ring widths. We will explore the excitonic $\mathrm{AB}$ effects in $3 \mathrm{D}$ type-I nanorings and investigate their dependence on the width of the nanoring, i.e., the non-1D character of the electron and hole motion. A similar model was recently successfully used to compute the exciton states in stacks of $(\mathrm{In}, \mathrm{Ga}) \mathrm{As} / \mathrm{GaAs}$ nanorings in the absence of a magnetic field. ${ }^{22}$

The paper is organized as follows. Sec. II describes our theoretical approach to compute the electronic structure of the electrons, holes, and excitons. The numerical results are presented and explained in Sec. III. Our conclusions are given in Sec. IV.

\section{THE THEORETICAL MODEL}

\section{A. The exciton Hamiltonian}

Both the (In,Ga)As/GaAs and $\mathrm{GaAs} /(\mathrm{Al}, \mathrm{Ga}) \mathrm{As}$ nanorings are made of sufficiently large band-gap semiconductors, and thus the single-band effective mass model,

$$
H=T+H_{Z}+V_{\text {eff }},
$$

can be used to compute the single-particle states in the conduction band. Here $T$ denotes the kinetic part of the Hamiltonian, $H_{Z}$ is the Zeeman term, and $V_{\text {eff }}$ is the effective potential which takes into account both the band offset between the ring and the matrix semiconductor and the influence of strain. For our axially symmetric rings, the use of cylindrical coordinates $\varphi, \rho$, and $z$ is appropriate.

Tetrahedral deformation of the crystal lattice due to strain in the (In, Ga)As/GaAs nanorings makes the effective potential well for the heavy holes deeper than the one for the light holes. Hence, the exciton ground state is mainly of heavy-hole character, which justifies the use of only the single-band effective-mass Hamiltonian to compute the electron and hole states in these nanorings. On the other hand, the use of the single-band model for the unstrained $\mathrm{GaAs} /(\mathrm{Al}, \mathrm{Ga}) \mathrm{As}$ nanorings is more limited. Yet, because of the larger effective mass, exciton wave functions are dominated by the heavy-hole envelope functions. Therefore, we use the single-band model for the heavy-hole states in both analyzed nanorings.

The strain distribution in the (In,Ga)As/GaAs nanorings is obtained from the 3D continuum mechanical model, following Ref. 21. For that purpose, a nonuniform 3D mesh is adopted. The computed strain distribution is incorporated in the effective potential with the assumption of axial symmetry by averaging the strain tensor components over the polar angle. ${ }^{23}$ Therefore, the effective potentials in the conduction and heavy-hole band depend only on $\rho$ and $z$,

$$
V_{\text {eff }}(\rho, z)=V_{\text {off }}(\rho, z)+V_{\text {str }}(\rho, z),
$$

where $V_{\text {off }}$ denotes the potential due to band offset, and $V_{\text {str }}$ is the strain-dependent effective potential. For the 
conduction-band electron

$$
V_{\mathrm{str}, e}=a_{c}\left(\varepsilon_{x x}+\varepsilon_{y y}+\varepsilon_{z z}\right),
$$

and for the heavy hole

$$
V_{\mathrm{str}, h}=-\left(a_{v}+\frac{b}{2}\right)\left(\varepsilon_{x x}+\varepsilon_{y y}\right)-\left(a_{v}-b\right) \varepsilon_{z z} .
$$

Here, $a_{c}, a_{v}$, and $b$ denote the deformation potentials, whereas $\varepsilon_{x x}, \varepsilon_{y y}$, and $\varepsilon_{z z}$ are the diagonal components of the strain tensor.

The kinetic part of the Hamiltonian is written in the symmetric gauge

$$
\begin{aligned}
T= & -\frac{\hbar^{2}}{2 m_{\|}} \frac{\partial^{2}}{\partial \rho^{2}}-\frac{\hbar^{2}}{2 m_{\|}} \frac{1}{\rho} \frac{\partial}{\partial \rho}-\frac{\hbar^{2}}{2 m_{\|}} \frac{1}{\rho^{2}} \frac{\partial^{2}}{\partial \varphi^{2}} \\
& -\frac{\hbar^{2}}{2} \frac{\partial}{\partial \rho}\left(\frac{1}{m_{\|}}\right) \frac{\partial}{\partial \rho} \\
& -\frac{\hbar^{2}}{2 m_{z}} \frac{\partial^{2}}{\partial z^{2}}-\frac{\hbar^{2}}{2} \frac{\partial}{\partial z}\left(\frac{1}{m_{z}}\right) \frac{\partial}{\partial z} \\
& +\frac{\hbar^{2}}{2 m_{\|}} \frac{1}{4 l_{c}^{4}} \rho^{2}-\frac{\hbar^{2}}{2 m_{\|}} \frac{i}{l_{c}^{2}} \frac{\partial}{\partial \varphi},
\end{aligned}
$$

where $l_{c}=(\hbar / e B)^{1 / 2}$ is the magnetic length, and $m_{\|}$and $m_{z}$ in Eq. (5) are the effective masses in the $x y$ plane and along the $z$ direction, respectively. $m_{\|}=m_{z}$ for the electron in the conduction band, while for the heavy hole $m_{\|}$and $m_{z}$ are extracted from the diagonal approximation of the multiband Luttinger-Kohn model. ${ }^{23,24}$

The Zeeman term is given by

$$
\begin{gathered}
H_{Z, e}= \pm g_{\text {eff }} \mu_{B} B / 2, \\
H_{Z, h}=\mp 3 \kappa \mu_{B} B,
\end{gathered}
$$

for the electrons and heavy holes, respectively. Here, $\mu_{B}$ is the Bohr magneton, $g_{\text {eff }}$ is the effective Landé $g$ factor, $\kappa$ is the Luttinger parameter describing the Zeeman splitting of the hole states, and the upper (lower) sign in Eqs. (6) and (7) refers to the spin-up (spin-down) state.

In order to solve the single-band effective-mass Schrödinger equation, $H \Psi=E \Psi$, the Galerkin form of the finite element method is employed. Our calculations rely on

$$
\int_{V}[u \mathbf{\nabla} \mathbf{A}+(\mathbf{A} \cdot \nabla) u] d \mathbf{r}=\oint_{S} u \mathbf{A} \cdot d \mathbf{S},
$$

where $V$ denotes the solution domain, $u$ is an arbitrary scalar function, $\mathbf{A}$ is an arbitrary vector-valued function, and $S$ is the boundary of $V$. When applied to the Hamiltonian (1), Eq. (8) gives

$$
\begin{aligned}
\int_{V} u\left(\tilde{T}+\tilde{H}_{Z}+\tilde{V}_{\mathrm{eff}}\right) \Psi d \mathbf{r} \\
=\int_{V} \frac{m_{0}}{m_{\|}}\left(\frac{1}{\rho^{2}} \frac{\partial u}{\partial \varphi} \frac{\partial \Psi}{\partial \varphi}+\frac{\partial u}{\partial \rho} \frac{\partial \Psi}{\partial \rho}\right) d \mathbf{r} \\
\quad+\int_{V} \frac{m_{0}}{m_{z}}\left(\frac{\partial u}{\partial z} \frac{\partial \Psi}{\partial z}\right) d \mathbf{r}+\int_{V} u \tilde{H}_{B}^{\prime} \Psi d \mathbf{r}+\int_{V} u \tilde{V} \Psi d \mathbf{r} .
\end{aligned}
$$

Here, $\tilde{T}, \tilde{H}_{Z}$, and $\tilde{V}_{\text {eff }}$, are $T, H_{Z}$, and $V_{\text {eff }}$ divided by $\hbar^{2} /\left(2 m_{0}\right)$, respectively. Furthermore, $\tilde{H}_{B}^{\prime}=H_{B}^{\prime} /\left(\hbar^{2} / 2 m_{0}\right)$, where $H_{B}^{\prime}$ are the last two terms of Eq. (5).

The single-particle Hamiltonian is axially symmetric, and therefore the projection of the orbital quantum number on the $z$ axis $L_{z}=l \hbar$ is a good quantum number for both the electron and hole states. The single-particle states are denoted by $n l^{\sigma}$, where $n$ denotes the principal quantum number, and $\sigma$ is a parity of the state with respect to $z$. They could additionally be labeled by spin, but we are interested in the (electron) spin-up states in the two bands whose eigenenergies are lower than the energies of the spin-down states. The wave function of the $n l^{\sigma}$ state is written as $\Psi_{l n}^{\sigma}$, and the eigenenergy as $E_{l n}^{\sigma}$. The energies of the electron and hole ground states, whose orbital momentum varies with $B$, are denoted by the symbols $E_{e}^{(1)}$ and $E_{h}^{(1)}$.

Because of axial symmetry, the single-particle wave function of the $n l^{\sigma}$ state can be written as

$$
\Psi_{l n}^{\sigma}(\varphi, \rho, z)=\frac{1}{\sqrt{2 \pi}} e^{i l \varphi} \psi_{\ln }^{\sigma}(\rho, z),
$$

where $\psi_{\ln }^{\sigma}(\rho, z)$ is expanded in products of the first-order shape functions

$$
\psi_{l n}^{\sigma}(\rho, z)=\sum_{j k} c_{j k} f_{j}(\rho) f_{k}(z),
$$

which are labeled by the mesh points, $j$ and $k$. On the master element $[-1,1]$, the first-order shape function has the form

$$
f(\xi)=\frac{1 \pm \xi}{2}
$$

Equation (11) leads to the generalized eigenvalue problem

$$
H c=E S c .
$$

The matrix elements of $H$ and $S$ are given by $H_{i j}=\left\langle f_{i}|H| f_{j}\right\rangle$ and $S_{i j}=\left\langle f_{i} \mid f_{j}\right\rangle$.

The exciton states are extracted from the equation

$$
H_{x} \Psi_{x}=\left(H_{e}+H_{h}+V_{C}\right) \Psi_{x}=E_{x} \Psi_{x},
$$

where $H_{x}$ denotes the exciton Hamiltonian, $E_{x}$ is the exciton eigenenergy, $\Psi_{x}=\Psi_{x}\left(\mathbf{r}_{e}, \mathbf{r}_{h}\right)$ is the exciton wave function, $H_{e}$ and $H_{h}$ are the electron and the hole Hamiltonian, respectively, and $V_{C}$ is the Coulomb potential,

$$
V_{C}\left(\mathbf{r}_{e}, \mathbf{r}_{h}\right)=-\frac{e^{2}}{4 \pi \epsilon_{s} \epsilon_{0} \sqrt{\rho_{x}^{2}+\left(z_{e}-z_{h}\right)^{2}}} .
$$

Here $\epsilon_{0}$ is the vacuum permittivity, $\epsilon_{s}$ is the relative permittivity of the material inside the ring, $z_{e}$ and $z_{h}$ are the values of the $z$ coordinate of the electron and the hole, respectively, whereas $\rho_{x}$ denotes the projection of the distance between the electron and the hole on the $x y$ plane,

$$
\rho_{x}=\left[\rho_{e}^{2}+\rho_{h}^{2}-2 \rho_{e} \rho_{h} \cos \left(\varphi_{e}-\varphi_{h}\right)\right]^{1 / 2} .
$$

From now on, $\rho_{x}$ will be referred as the in-plane distance between the electron and the hole.

Let us briefly examine which quantum numbers label the exciton states. The in-plane distance $\rho_{x}$ does not depend on the polar angles of the electron and the hole, $\varphi_{e}$ and $\varphi_{h}$, separately, but on the difference $\varphi_{e}-\varphi_{h}$. It implies that rotation of the exciton over the $z$ axis by an arbitrary angle does not 
affect the Coulomb interaction, and consequently the orbital momentum $L=l_{e}+l_{h}$ is a good quantum number for the exciton. Furthermore, $H_{x}$ possesses inversion symmetry with respect to simultaneous reversal of the $z_{e}$ and $z_{h}$ coordinates. Therefore, the exciton parity $\sigma_{x}$ is a good quantum number. The even and odd exciton states, $\sigma_{x}=+$ and $\sigma_{x}=-$, respectively, are composed of the electron and hole states of equal and opposite parity, respectively. For the given exciton and electron parities, $\sigma_{x}$ and $\sigma_{e}$, respectively, the hole parity $\sigma_{h}=\sigma_{h}\left(\sigma_{x}, \sigma_{e}\right)$ has the following values:

$$
\begin{aligned}
& \sigma_{h}(+,+)=+, \quad \sigma_{h}(+,-)=-, \\
& \sigma_{h}(-,+)=-, \quad \sigma_{h}(-,-)=+.
\end{aligned}
$$

The exciton states are arranged in spin quartets $[\uparrow \uparrow, \uparrow \downarrow$, $\downarrow \uparrow, \downarrow \downarrow]$, where the first (second) arrow indicates the spin of the conduction (valence) band state. The Zeeman terms in Eq. (14) remove the degeneracy between the spin quartets so that the $\uparrow \uparrow$ exciton state has the lowest energy among their counterparts. Furthermore, energies of the odd exciton states are higher by a few tens of meV from those of the even exciton states. Therefore, only even exciton states of the spin-up electron and spin-up hole are presented and discussed in Sec. III. The exciton states are denoted by $n L^{\sigma_{x}}$, and the exciton eigenenergies by $E_{x n L}^{\sigma_{x}}$, where $n$ denotes the principal quantum number. For the energies of the $n S^{+}$states we use the abbreviated symbol $E_{x}^{(n)}$.

In our exact diagonalization approach, we expand the exciton wave function as follows:

$$
\Psi_{x}=\sum_{\sigma_{e}} \sum_{l_{e}} \sum_{n_{e}} \sum_{n_{h}} c_{l_{e}, n_{e}, n_{h}}^{\sigma_{e}} \Psi_{e h\left(l_{e}, n_{e}\right),\left(l_{h}, n_{h}\right)}^{\sigma_{e}, \sigma_{h}},
$$

where $l_{h}=L-l_{e}, \sigma_{h}=\sigma_{h}\left(\sigma_{x}, \sigma_{e}\right)$, and $\Psi_{e h}$ denotes the electron-hole wave function,

$$
\Psi_{e h\left(l_{e}, n_{e}\right),\left(l_{h}, n_{h}\right)}^{\sigma_{e}, \sigma_{h}}=\Psi_{l_{e}, n_{e}}^{\sigma_{e}}\left(\mathbf{r}_{e}\right) \Psi_{l_{h}, n_{h}}^{\sigma_{h}}\left(\mathbf{r}_{h}\right) .
$$

The exciton Hamiltonian now reads

$$
\begin{aligned}
H_{x}= & \left(E_{l_{e}, n_{e}}^{\sigma_{e}}+E_{l_{h}, n_{h}}^{\sigma_{h}}\right) \delta_{\sigma_{e}^{\prime}, \sigma_{e}} \delta_{l_{e}^{\prime}, l_{e}} \delta_{n_{e}^{\prime}, n_{e}} \delta_{n_{h}^{\prime}, n_{h}} \\
& +\sum_{\sigma_{e}} \sum_{l_{e}} \sum_{n_{e}} \sum_{n_{h}}\left\langle\Psi_{l_{e}^{\prime}, n_{e}^{\prime}}^{\sigma_{e}^{\prime}} \Psi_{l_{h}^{\prime}, n_{h}^{\prime}}^{\sigma_{h}^{\prime}}\left|V_{C}\right| \Psi_{l_{e}, n_{e}}^{\sigma_{e}} \Psi_{l_{h}, n_{h}}^{\sigma_{h}}\right\rangle,
\end{aligned}
$$

where $\delta$ denotes the Kronecker delta and $E_{g m}$ is the energy gap in the GaAs matrix. A straightforward calculation of the Coulomb matrix element gives

$$
\begin{aligned}
& \left\langle\Psi_{l_{e}^{\prime}, n_{e}^{\prime}}^{\sigma_{e}^{\prime}} \Psi_{l_{h}^{\prime}, n_{h}^{\prime}}^{\sigma_{h}^{\prime}}\left|V_{C}\right| \Psi_{l_{e}, n_{e}}^{\sigma_{e}} \Psi_{l_{h}, n_{h}}^{\sigma_{h}}\right\rangle=\frac{1}{4 \pi^{2}} \frac{e^{2}}{\epsilon \epsilon_{0}} \delta_{l_{e}^{\prime}+l_{h}^{\prime}, l_{e}+l_{h}} \\
& \quad \times \int_{0}^{\infty} d k_{\|} k_{\|} \int_{-\infty}^{+\infty} d k_{z} \frac{1}{k_{\|}^{2}+k_{z}^{2}} \mathcal{F}_{e}\left(k_{\|},-k_{z}\right) \mathcal{F}_{h}\left(k_{\|}, k_{z}\right)
\end{aligned}
$$

where $k_{\|}$and $k_{z}$ denote the in-plane and the $z$ component of the wave vector in Fourier space, respectively. $\mathcal{F}_{e}$ is the two-dimensional transform given by

$$
\mathcal{F}_{e}\left(k_{\|}, k_{z}\right)=\int_{0}^{\infty} \rho d \rho \int_{-\infty}^{+\infty} d z \psi_{l_{e}^{\prime}, n_{e}^{\prime}}^{\sigma_{e}^{\prime} *} \psi_{l_{e}, n_{e}}^{\sigma_{e}} J_{\left|l_{e}^{\prime}-l_{e}\right|}\left(k_{\|} \rho\right) e^{i k_{z} z},
$$

with $J_{l}(x)$ being the Bessel function of the first kind.
As a figure of merit of the exciton, we compute the average exciton in-plane radius $R_{\|}$, whose square is given by

$$
R_{\|}^{2}=\left\langle\rho_{x}^{2}\right\rangle=\int_{V_{e}} d \mathbf{r}_{e} \int_{V_{h}} \rho_{x}^{2}\left(\mathbf{r}_{e}, \mathbf{r}_{h}\right)\left|\Psi_{x}\left(\mathbf{r}_{e}, \mathbf{r}_{h}\right)\right|^{2} d \mathbf{r}_{h},
$$

where $\rho_{x}\left(\mathbf{r}_{e}, \mathbf{r}_{h}\right)$ is given by Eq. (16). Replacing the singleparticle wave function with the form in Eq. (10), and taking into account parity, results in

$$
\begin{aligned}
R_{\|}^{2}= & \sum_{\sigma_{e}} \sum_{l_{e}} \sum_{n_{e}^{\prime}, n_{e}} \sum_{n_{h}^{\prime}, n_{h}} c_{l_{e}, n_{e}^{\prime}, n_{h}^{\prime}}^{\sigma_{e} *} c_{l_{e}, n_{e}, n_{h}}^{\sigma_{e}} \\
& \times\left[\left\langle l_{e}, n_{e}^{\prime}\left|\rho_{e}^{2}\right| l_{e}, n_{e}\right\rangle \delta_{l_{h}^{\prime}, l_{h}}+\delta_{l_{e}^{\prime}, l_{e}}\left\langle l_{h}, n_{h}^{\prime}\left|\rho_{h}^{2}\right| l_{h}, n_{h}\right\rangle\right] \\
& -c_{l_{e}-1, n_{e}^{\prime}, n_{h}^{\prime}}^{\sigma_{e}} c_{l_{e}, n_{e}, n_{h}}^{\sigma_{e}} \\
& \times\left\langle l_{e}-1, n_{e}^{\prime}\left|\rho_{e}\right| l_{e}, n_{e}\right\rangle\left\langle l_{h}+1, n_{h}^{\prime}\left|\rho_{h}\right| l_{h}, n_{h}\right\rangle \\
& -c_{l_{e}+1, n_{e}^{\prime}, n_{h}^{\prime}}^{\sigma_{e} *} c_{l_{e}, n_{e}, n_{h}}^{\sigma_{e}} \\
& \times\left\langle l_{e}+1, n_{e}^{\prime}\left|\rho_{e}\right| l_{e}, n_{e}\right\rangle\left\langle l_{h}-1, n_{h}^{\prime}\left|\rho_{h}\right| l_{h}, n_{h}\right\rangle,
\end{aligned}
$$

where $l_{h}=L-l_{e}$. The matrix elements in Eq. (24),

$$
\left\langle l^{\prime}, n^{\prime}\left|\rho^{k}\right| l, n\right\rangle=\int_{-H / 2}^{H / 2} d z \int_{0}^{R} \rho^{k} \psi_{l^{\prime} n^{\prime}}^{*}(\rho, z) \psi_{l n}(\rho, z) \rho d \rho,
$$

are computed numerically for $k=0,1,2$ on the solution domain of radius $R$ and height $H$.

In addition to $R_{\|}$, a few other parameters of the exciton states are computed: (1) the binding energy of the exciton

$$
E_{b}=E_{e}^{(1)}+E_{h}^{(1)}-E_{x}^{(1)}
$$

(2) the contribution of the electron orbital momentum $l_{e}$ to the exciton wave function

$$
p_{l_{e}}=\sum_{\sigma_{e}} \sum_{n_{e}} \sum_{n_{h}}\left|c_{l_{e}, n_{e}, n_{h}}^{\sigma_{e}}\right|^{2}
$$

and (3) the conditional probability density (CPD)

$$
P\left(\mathbf{r}_{h} \mid \mathbf{r}_{e 0}\right)=\frac{\left|\Psi_{x}\left(\mathbf{r}_{e 0}, \mathbf{r}_{h}\right)\right|^{2}}{\int_{V_{h}} \mid \Psi_{x}\left(\mathbf{r}_{e 0},\left.\mathbf{r}_{h}\right|^{2} d \mathbf{r}_{h}\right.} .
$$

The last parameter is the probability to find the hole at $\mathbf{r}_{h}$ under the condition that the electron is located at $\mathbf{r}_{e 0}=\left(\varphi_{e 0}, \rho_{e 0}, z_{e 0}\right)$. The exciton wave function depends on the difference $\varphi_{e}-\varphi_{h}$, and therefore the shape of the CPD does not depend on the choice of $\varphi_{e 0}$, and thus we may take $\varphi_{e 0}=0$. The other two coordinates $\rho_{e 0}$ and $z_{e 0}$ are chosen to correspond to the position of the maximum of the probability density of the exciton state $\left|\Psi_{x}\right|^{2}$. It appeared that the value $\rho_{e 0}$ is close to the average radius of the ring $\left(R_{1}+R_{2}\right) / 2$, whereas $z_{e 0}=0$.

\section{B. The two-level exciton Hamiltonian}

The exact diagonalization approach presented in the previous section produces relatively large Hamiltonian matrices and lacks a simple parametrization of the $E_{x}$ dependence on $B$. It turns out that the amplitude of the excitonic $\mathrm{AB}$ oscillations depends on how efficiently $1 S^{+}$and $2 S^{+}$states couple with varying magnetic field. Therefore we construct a simple model to approximately describe coupling between these states. It has the form of a $2 \times 2$ matrix and is derived by means of 
Löwdin perturbation theory applied to the exciton Hamiltonian of Eq. (20). This model is restricted to the range $0 \leqslant B \leqslant B_{1}$, where the $1 S^{+}$and $2 S^{+}$exciton states are mainly composed of the $\Psi_{(0,1)_{e}}^{+} \Psi_{(0,1)_{h}}^{+} \equiv|0,0\rangle$ and $\Psi_{(-1,1)_{e}}^{+} \Psi_{(+1,1)_{h}}^{+} \equiv|-1,+1\rangle$ electron-hole states. The latter states form the basis to expand the exciton wave function, which yields

$$
H_{x 0}=\left[\begin{array}{cc}
E_{e h(0,0)} & t_{0} \\
t_{0} & E_{e h(-1,+1)}
\end{array}\right] .
$$

Here,

$$
E_{e h(0,0)}=E_{e(0,1)}+E_{h(0,1)}
$$

and

$$
E_{e h(-1,+1)}=E_{e(-1,1)}+E_{h(+1,1)}
$$

are the energies of the electron-hole states, and $t_{0}$ denotes the transfer integral due to the Coulomb interaction between the two electron-hole states in the basis. The other electronhole states have higher energies than the two basis states, and their contribution to the exciton states is approximately taken into account by the Löwdin perturbation expansion

$$
H_{x, i j}^{\prime}=\sum_{k=3}^{N} \frac{H_{x, i k} H_{x, k j}}{E-H_{x, k k}} .
$$

The value of the unperturbed energy $E$ is estimated as

$$
E=\min \left(E_{e h(0,0)}, E_{e h(-1,+1)}\right),
$$

and $N$ is the total number of electron-hole states for $L=0$ in the expansion in Eq. (18). Hence, the approximate Hamiltonian has the form

$$
\tilde{H}_{x}=H_{x 0}+H_{x}^{\prime}=\left[\begin{array}{cc}
\tilde{E}_{(0,0)} & t_{B} \\
t_{B} & \tilde{E}_{(-1,+1)}
\end{array}\right] .
$$

The diagonal elements in this matrix are given by

$$
\tilde{E}_{(0,0)}=E_{e h(0,0)}+\Delta E_{(0,0)}
$$

and

$$
\tilde{E}_{(-1,+1)}=E_{e h(-1,+1)}+\Delta E_{(-1,+1)},
$$

where $\Delta E_{(0,0)}$ and $\Delta E_{(-1,+1)}$ are due to coupling with the electron-hole states outside the basis. The transfer integral $t_{B}$ in Eq. (34) essentially describes how the $|0,0\rangle$ and $|-1,+1\rangle$ states couple due to the Coulomb interaction in the $1 S^{+}$and $2 S^{+}$states. Because $\left|t_{B}\right| \ll \tilde{E}_{e h(0,0)}$ and $\left|t_{B}\right| \ll \tilde{E}_{e h(-1,+1)}$, the Hamiltonian (34) has the form of a tight-binding approximation to the exciton Hamiltonian.

The dependence of $t_{B}$ on $B$ is determined numerically, and thus $\tilde{H}_{x} \tilde{c}_{x}=E_{x} \tilde{c}_{x}$ does not have an analytical solution. Nevertheless, we note that the single-particle energy levels depend approximately quadratically on $B$. Also, $\left|\Delta E_{(0,0)}\right| \ll$ $E_{(0,0)}$, and $\left|\Delta E_{(-1,+1)}\right| \ll E_{(-1,+1)}$; therefore the diagonal matrix elements of $\tilde{H}_{x}$ are approximated as

$$
\tilde{E}_{(0,0)}(B) \approx E_{(0,0) 0}+a_{0}\left(B-B_{(0,0) 0}\right)^{2}
$$

and

$$
\tilde{E}_{(-1,+1)}(B) \approx E_{(-1,+1) 0}+a_{1}\left(B-B_{(-1,+1) 0}\right)^{2},
$$

where $a_{0}, a_{1}, B_{(0,0) 0}, B_{(-1,+1) 0}, E_{(0,0) 0}$, and $E_{(-1,+1) 0}$ are constants. The energies of the electron-hole states $E_{e h(0,0)}$ and $E_{e h(-1,+1)}$ cross close to $B_{1} / 2$. Because $\left|\Delta E_{0,0}\right| \ll E_{e h(0,0)}$ and $\left|\Delta E_{-1,+1}\right| \ll E_{e h(-1,+1)}, \tilde{E}_{(0,0)}$ and $\tilde{E}_{(-1,+1)}$ also cross close to $B_{1} / 2$, i.e., at $B=B_{c} \approx B_{1} / 2$.

Moreover, the transfer integral $t_{B}$ does not vary much with $B$, which will be illustrated below. Because the dependence of the exciton energy levels with $B$ around $B_{1} / 2$ is of interest to us, $t_{B}$ in Eq. (34) might be replaced with $t=t_{B}\left(B_{c}\right) \approx$ $t_{B}\left(B_{1} / 2\right)$. Hence, the approximate form of the two-level model (TLM) reads

$$
\tilde{H}_{x} \approx\left[\begin{array}{cc}
\tilde{E}_{(0,0)} & t \\
t & \tilde{E}_{(-1,+1)}
\end{array}\right] .
$$

The solutions of the secular equation $\operatorname{det}\left(\tilde{H}_{x}-E_{x} I\right)=0$ are given by

$$
E_{x}^{ \pm}=f_{+} \pm \sqrt{f_{-}+t^{2}}
$$

where

$$
f_{ \pm}=\frac{\tilde{E}_{(-1,+1)} \pm \tilde{E}_{(0,0)}}{2} .
$$

The exciton energy levels given by Eq. (40) will be compared with the results from the above exact diagonalization approach. The advantage of the former is that oscillatory features of the $E_{x}(B)$ function are described only by a few parameters. Furthermore, these parameters could be adjusted to increase the amplitude of the oscillations in the exciton energy. For example, varying the dimensions of the ring and introducing mechanical strain in the structure could increase the overlap between the electron and hole wave functions, which affects the transfer integral and in turn the dependence of $E_{x}$ on $B$. When the magnitude of $t$ increases, coupling between the two exciton states computed by the TLM increases. It then leads to a decrease of the gap between the $E_{x}^{-}$and $E_{x}^{+}$states, and in turn the amplitude of the oscillations in the exciton ground-state energy increases.

\section{The oscillator strength for exciton recombination} by

The oscillator strength for exciton recombination is given

$$
f_{x}=\frac{2}{m_{0} E_{x}}\left|\left\langle u_{c 0}|\boldsymbol{\varepsilon} \cdot \mathbf{p}| u_{v 0}\right\rangle\right|^{2}|M|^{2} .
$$

Here, $\boldsymbol{\varepsilon}$ denotes the unit vector of polarization of out-coming light, $u_{c 0}$ and $u_{v 0}$ are the periodic parts of the Bloch functions of the electron in the conduction and valence band, respectively, $\mathbf{p}$ is the electron momentum, $E_{x}$ is the exciton energy, $m_{0}$ is the free-electron mass, and $M$ denotes the transition matrix element between the envelope functions, ${ }^{27}$

$$
M=\int_{V_{e}, V_{h}} \delta\left(\mathbf{r}_{e}-\mathbf{r}_{h}\right) \Psi_{x}\left(\mathbf{r}_{e}, \mathbf{r}_{h}\right) d \mathbf{r}_{e} d \mathbf{r}_{h} .
$$

For equal spins of the electron and the hole, and even parity of the exciton, only $L=l_{e}+l_{h}=0$ exciton states are bright; therefore

$$
M=\sum_{\sigma_{e}} \sum_{l_{e}} \sum_{n_{e}} \sum_{n_{h}} c_{l_{e}, n_{e}, n_{h}}^{\sigma_{e}}\left\langle l_{e}, n_{e} \mid-l_{e}, n_{h}\right\rangle .
$$


We assume light polarized along the $x$ direction, for which the matrix element squared between the zone center states is given by $^{25,26}$

$$
\left|\left\langle u_{c 0}\left|p_{x}\right| u_{v 0}\right\rangle\right|^{2}=\frac{m_{0}^{2} P^{2}}{2 \hbar^{2}},
$$

where $P$ denotes the Kane interband matrix element. When Eqs. (43)-(45) are inserted in Eq. (42), the expression for $f_{x}$ of a bright exciton state follows

$$
f_{x}=\frac{1}{2} \frac{E_{P}}{E_{x i}}\left|\sum_{\sigma_{e}} \sum_{l_{e}} \sum_{n_{e}} \sum_{n_{h}} c_{l_{e}, n_{e}, n_{h}}^{\sigma_{e}}\left\langle l_{e}, n_{e} \mid-l_{e}, n_{h}\right\rangle\right|^{2},
$$

where $E_{P}=2 m_{0} P^{2} / \hbar^{2}$. At finite temperature, the dark states are occupied with a finite probability. One defines the (dimensionless) photoluminescence intensity, ${ }^{15}$

$$
I_{P L}=\frac{\sum_{i} f_{x i} \exp \left(-E_{x i} / k_{B} T\right)}{\sum_{i} \exp \left(-E_{x i} / k_{B} T\right)},
$$

which takes into account that the exciton states, labeled by a single index $i$, are populated according to Boltzmann statistics.

\section{NUMERICAL RESULTS AND DISCUSSION}

We compute the exciton states in both strained (In,Ga)As/GaAs and unstrained $\mathrm{GaAs} /(\mathrm{Al}, \mathrm{Ga}) \mathrm{As}$ nanorings. The (In,Ga)As/GaAs nanorings have been recently fabricated and analyzed. ${ }^{18}$ X-STM analysis revealed they have nearly circular cross section with inner radius $R_{1}=8 \mathrm{~nm}$, outer radius $R_{2}=15 \mathrm{~nm}$, and height $h=4 \mathrm{~nm}$. Therefore, in our numerical calculations we took $R_{1}$ equals $8 \mathrm{~nm}, h=4 \mathrm{~nm}$, whereas the ring width $W$ is varied in the range from 2 to $22 \mathrm{~nm}$. We assumed that the mole fraction of InAs in the ring is $x=0.55$. $^{18}$ As previously noted, the GaAs/(Al,Ga)As nanorings have been produced by means of the droplet epitaxy technique. ${ }^{20}$ Their lateral dimensions are usually larger than the respective dimensions of the (In,Ga)As/GaAs nanorings. But, in order to explore how mechanical strain affects the magnetic field dependence of the exciton energy levels, the $\mathrm{GaAs} /(\mathrm{Al}, \mathrm{Ga}) \mathrm{As}$ nanorings are assumed to have the same dimensions as the (In,Ga)As/GaAs nanorings. The $(\mathrm{Al}, \mathrm{Ga}) \mathrm{As}$ alloy is assumed to contain $30 \%$ of AlAs.

The parameters of the band structure and elastic constants of (In,Ga)As, GaAs, and (Al,Ga)As are all taken from Ref. 24. The conduction-band offset amounts to $83 \%$ and $65 \%$ of the band-gap difference in the (In,Ga)As/GaAs and $\mathrm{GaAs} /(\mathrm{Al}, \mathrm{Ga}) \mathrm{As}$ system, respectively. ${ }^{24,28}$ Measurements of the Zeeman splitting of the energy levels in the (In,Ga)As/GaAs nanodots indicated that the energy-level splitting is much smaller than what is found in bulk (In,Ga)As and to be closer to bulk GaAs. ${ }^{29}$ Therefore, the effective Landé $g$ factor $g_{\text {eff }}$ and the Luttinger parameter $\kappa$ in both analyzed structures are assumed to be position independent and to be equal to the values in GaAs, -0.44 and 1.2, respectively. The basis for the exciton states is constructed from 6 even and 2 odd single-particle states for each $l\left(l_{e}\right.$ or $\left.l_{h}\right)$ in the range from -7 to +7 . We took temperature $T=1 \mathrm{~K}$.

The nonuniform mesh in the finite-element calculation of the single-particle states is constructed from 129 points along both the $\rho$ and $z$ direction. The expansion domain is
$H=200 \mathrm{~nm}$ high and its radius is $R=120 \mathrm{~nm}$. Such a large domain was needed because the effective potentials decrease slowly in the matrix as a result of a slow strain decay toward the zero value when the distance from the ring increases. ${ }^{22}$ We note that the effective potentials are not abrupt, yet they have large slope due to small size of employed quadrilateral elements in the vicinity of the ring boundary. We used the same mesh for both the (In,Ga)As/GaAs and GaAs/(Al,Ga)As nanorings.

\section{A. The exciton states in the strained nanorings}

The effective potentials in the conduction and valence bands of the $W=7 \mathrm{~nm}$ wide (In,Ga)As/GaAs nanoring along the $\rho$ direction are shown in Figs. 2(a) and 2(b), respectively. The effective potential well for the electron is deeper than the effective potential well for the heavy hole. On the other hand, the heavy hole is confined in a wide effective potential well, which is much deeper than the effective potential well for the light hole. It turns out that the energy levels of the light hole are pushed by strain further from the heavy-hole energy levels towards the continuum. Consequently, strain reduces mixing between the light holes and the heavy holes, ${ }^{23}$ which supports the use of the diagonal approximation of the Luttinger-Kohn model when computing the hole states in the

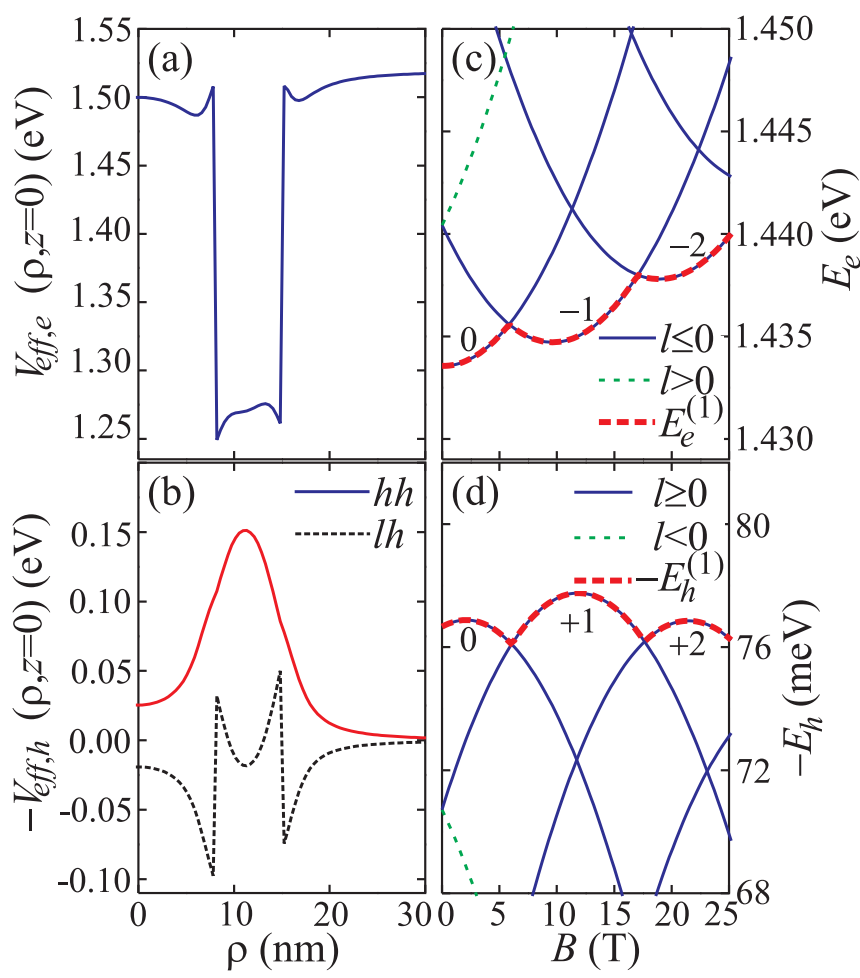

FIG. 2. (Color online) (a) The effective potential for the electron in the (In,Ga)As/GaAs nanoring along the radial axis for $z=0$. (b) The same for the heavy hole (solid line) and the light hole (dashed line). (c) The electron energy levels in the (In,Ga)As/GaAs nanoring as they vary with magnetic field. (d) Negative of the heavy-hole eigenenergies as function of magnetic field. The ground states $E_{e}^{(1)}$ and $E_{h}^{(1)}$ oscillate with magnetic field, and the orbital momentum changes, as indicated by numbers adjacent to the curves. The ring has an inner radius $R_{1}=8 \mathrm{~nm}$, a width $W=7 \mathrm{~nm}$, and a height $h=4 \mathrm{~nm}$. 

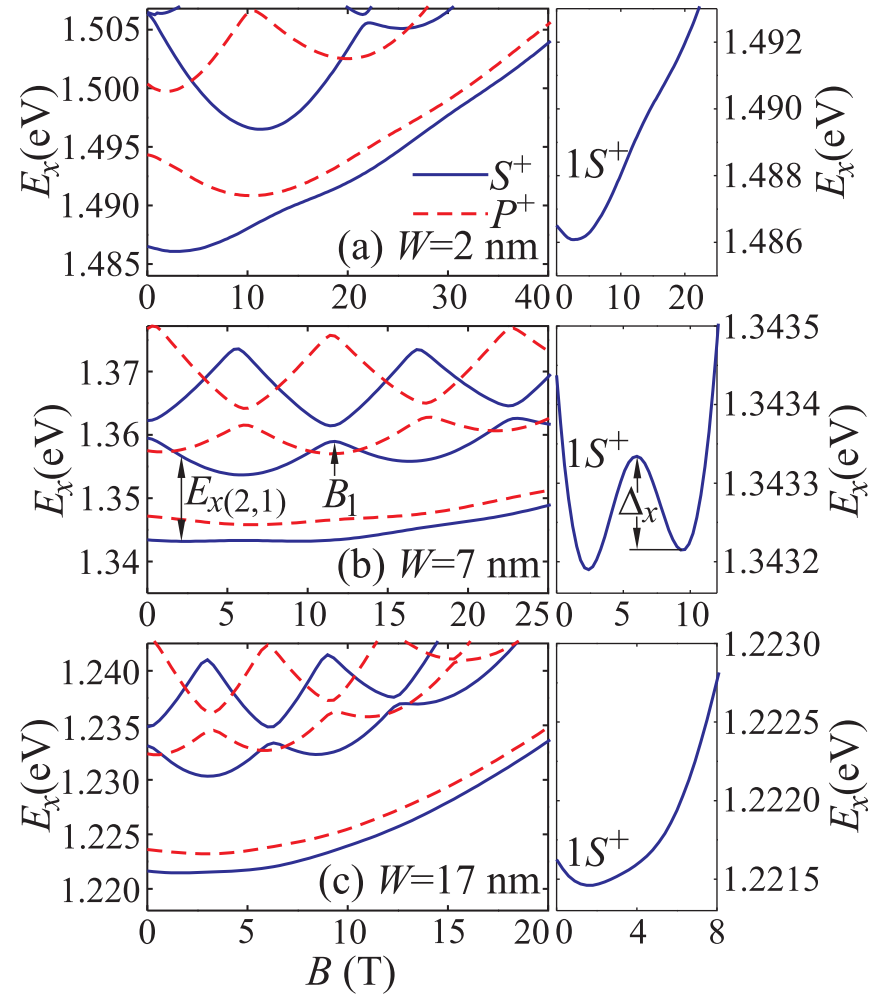

FIG. 3. (Color online) The lowest energy $L=0$ (solid lines) and $L=-1$ states (dashed lines) in (a) the $W=2 \mathrm{~nm}$, (b) the $W=$ $7 \mathrm{~nm}$, and (c) the $W=17 \mathrm{~nm}$ wide (In,Ga)As/GaAs nanoring. $B_{1}$ denotes the magnetic field value where $E_{x}^{(2)}$ has a maximum due to the anticrossing with the $E_{x}^{(3)}$ state. Right panel displays detailed views of the exciton ground-state energy at low magnetic fields.

(In,Ga)As/GaAs nanorings. Variations of the electron and hole states with magnetic field are shown in Figs. 2(c) and 2(d). Both the electron and hole energy levels show orbital momentum transitions, which take place at almost the same magnetic field values. Therefore, to a large extent we may infer that the orbital momentum of the exciton ground state is $L=l_{e}+l_{h}=0$ irrespective of the magnetic field value. The magnetic field where the $1 s^{+}$and $2 s^{+}$energy levels cross corresponds approximately to the condition that half the flux quantum $\left(\Phi=\Phi_{0}=h / 2 e\right)$ threads the 1D ring of the radius $\left(R_{1}+R_{2}\right) / 2$.

The exciton ground state is indeed $1 S^{+}$, as Figs. 3(a), 3(b), and 3(c) show for the $W=2, W=7$, and $W=17 \mathrm{~nm}$ wide (In,Ga)As/GaAs nanoring, respectively. Details of $E_{x}^{(1)}$ as a function of $B$ for the three cases are shown in the right panel of Fig. 3, and show clearly that the width of the ring affects the energy variation of the exciton ground state with magnetic field. While the case of the $W=17 \mathrm{~nm}$ wide ring, Fig. 3(c), does not clearly demonstrate oscillations in $E_{x}^{(1)}$, they become evident in the $W=2$ and $7 \mathrm{~nm}$ wide rings [see Figs. 3(a) and 3(b)]. The first minimum of $E_{x}^{(1)}$ in Figs. 3(a)-3(c) arises from anticrossing with the $2 S^{+}$state and is affected by the Zeeman splitting at low magnetic field, when the diamagnetic shift is small. Out of the three curves in the right panel of Fig. 3, only $E_{x}^{(1)}$ of the $7 \mathrm{~nm}$ wide ring exhibits two minima. By comparing Figs. 2 and 3, we see that the anticrossings of the exciton state take place close to the orbital momentum transitions of the single-particle states. The energy difference between $E_{x}^{(2)}$ and $E_{x}^{(1)}$, i.e., $E_{x(2,1)}=E_{x}^{(2)}-E_{x}^{(1)}$, is explicitly indicated in Fig. 3(b). The other parameter is $\Delta_{x}$, the difference between the first maximum and the second minimum of the $E_{x}^{(1)}(B)$ curve, which is indicated in the right panel of Fig. 3(b). Figures 3(a) and 3(c) indicate that the effect of the anticrossing in the $2 \mathrm{~nm}$ and $17 \mathrm{~nm}$ wide rings is so strong that small or no $\mathrm{AB}$ oscillations are visible. For the intermediate case, shown in Fig. 3(b), the effect of anticrossing is relatively small, and therefore a second minimum appears in the $E_{x}^{(1)}$ dependence on $B$. Moreover, irrespective of the ring width, quite large oscillations, with amplitude of the order of $10 \mathrm{meV}$, exist in the higher exciton energy levels. The value of magnetic field where the $2 S^{+}$and $3 S^{+}$states anticross, which is denoted by $B_{1}$, is explicitly indicated in Fig. 3(b). The observed anticrossings are modeled with the TLM, and those results will be presented and discussed in the following section.

Figure 4(a) shows the variation of $E_{x(2,1)}$ with $B$ in the $W=7 \mathrm{~nm}$ wide (In,Ga)As/GaAs nanoring. It is oscillatory with the minima corresponding to anticrossings between the $1 S^{+}$and $2 S^{+}$states. The ratio of magnetic field values where these minima take place is close to $1: 3: 5: \ldots$. which is the exact order of the single-particle orbital momentum transitions in 1D

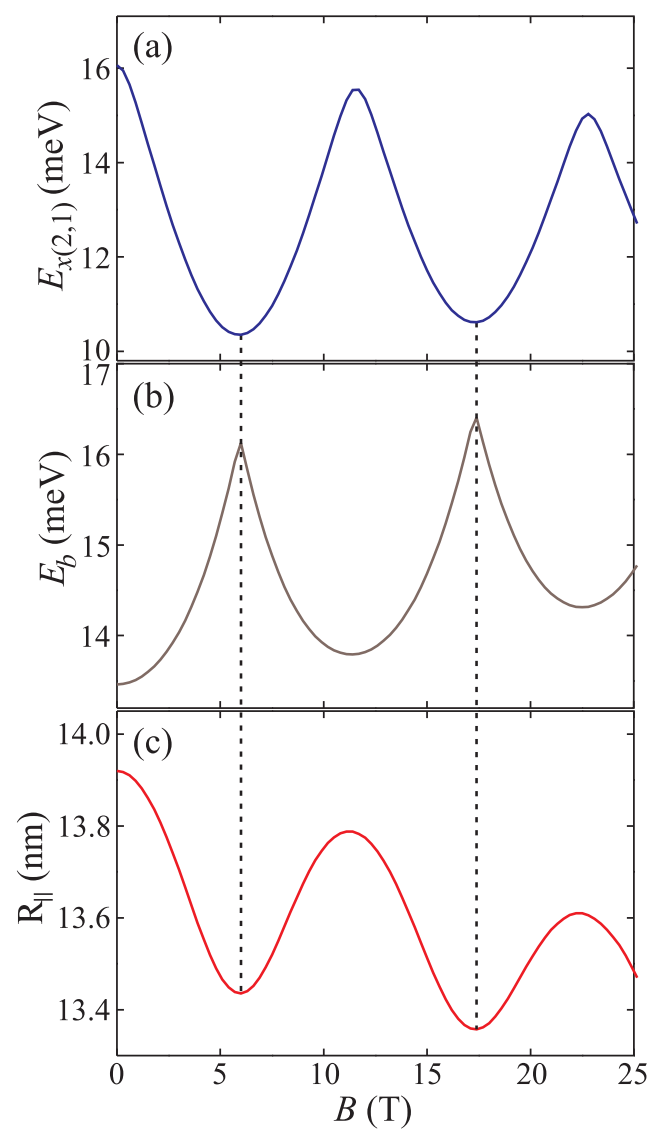

FIG. 4. (Color online) (a) The difference between the two lowest energy $L=0$ exciton states, $E_{x(2,1)}$, (b) the binding energy of the exciton ground state $E_{b}$, and (c) the average in-plane radius $R_{\|}$as they vary with $B$ in the $W=7 \mathrm{~nm}$ wide (In,Ga)As/GaAs nanoring. The minima in (a) and (c) correspond to maxima in (b), which is indicated by the dashed vertical lines. 
rings. ${ }^{19}$ The radius of the equivalent $1 \mathrm{D}$ ring estimated from the flux quantum $\Phi_{0}=h / e$ and the magnetic field interval between two minima $\Delta B$ in Fig. 4(a) is $R_{1 \mathrm{D}}=(h / e \pi \Delta B)^{1 / 2}$ $=10.7 \mathrm{~nm}$, which is close to the average of the inner and outer radius of the ring, $\left(R_{1}+R_{2}\right) / 2=11.5 \mathrm{~nm}$. Each minimum of $E_{x(2,1)}$ corresponds to a maximum of the binding energy $E_{b}$, as shown in Fig. 4(b). Furthermore, due to the nonsmooth variation of $E_{h}^{(1)}$ and $E_{e}^{(1)}$ (see Fig. 2), $E_{b}$ exhibits spikes at anticrossings. When $E_{b}$ is large, the Coulomb interaction is large, and the electron and the hole are bound close to each other, as demonstrated in Fig. 4(c). Oscillations in $R_{\|}$are clearly observed in Fig. 4(c), although the amplitude of these oscillations is not large.

The positions of the anticrossings in Fig. 3 are associated with the crossings between different $p_{l_{e}}(B)$ curves, as Figs. 5(a) and 5(b) demonstrate for the $1 S^{+}$and $2 S^{+}$state, respectively. For $B<B_{c} \approx B_{1} / 2$ the largest contribution to the $1 S^{+}$state arises from the $l_{e}=0$ states [see Fig. 5(a)], and the $l_{e}=-1$ electron wave functions dominate in the $1 S^{+}$ exciton state for $B_{c}<B<B_{1}$. In contrast, the $2 S^{+}$state is mainly composed of the $l_{e}=-1$ states for $B<B_{c}$, whereas the $l_{e}=0$ mostly contribute to this state for $B_{c}<B<B_{1}$. Hence, when two exciton states anticross, the composition of the exciton wave function changes. Furthermore, the $p_{0}(B)$ curve crosses the $p_{-2}(B)$ curve at $B=B_{1}$.
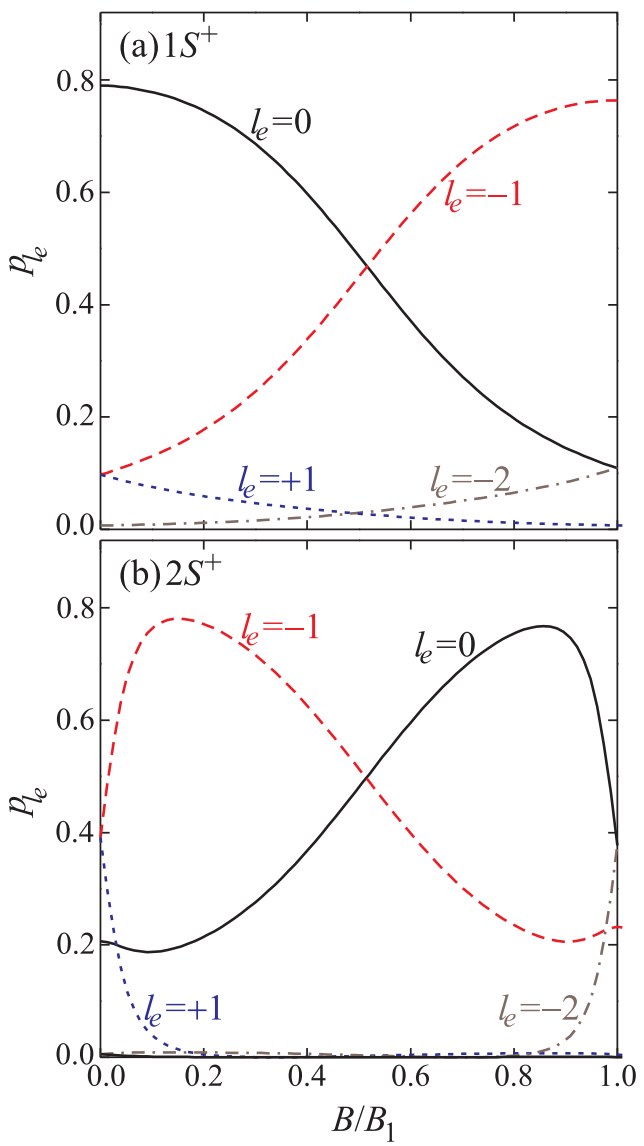

FIG. 5. (Color online) The contribution of the $l_{e}=-2,-1,0$, and +1 electron states to (a) the $1 S^{+}$and (b) the $2 S^{+}$states in the $W=7 \mathrm{~nm}$ wide (In,Ga)As/GaAs nanoring.

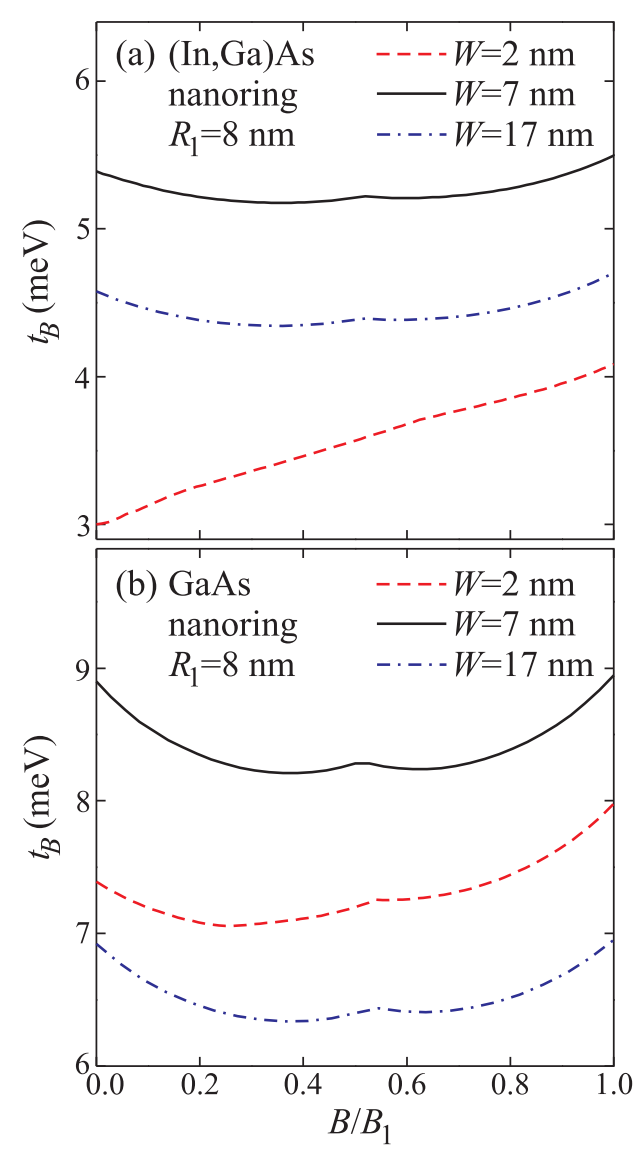

FIG. 6. (Color online) The dependence of the transfer integral $t_{B}$ on the magnetic field in (a) the (In,Ga)As/GaAs and (b) the $\mathrm{GaAs} /(\mathrm{Al}, \mathrm{Ga})$ As nanorings.

\section{B. Comparisons between strained and unstrained nanorings}

An important parameter given by Eq. (34) in the TLM of the excitonic Hamiltonian in this model is the transfer integral $t_{B}$. Figures 6(a) and 6(b) show the dependence of $t_{B}$ on $B$ in the range $\left[0, B_{1}\right]$. Due to the crossings between the single particle states, $t_{B}$ vs $B$ exhibits a kink at $B=B_{c}$. Also, it is of the order of a few meV in both structures, and varies by less than $1 \mathrm{meV}$; hence the approximation $t(B) \approx t\left(B_{C}\right) \equiv t_{B}$, employed to derive the TLM, is justified. The largest $t_{B}$ is found in the $W=7 \mathrm{~nm}$ wide ring, while comparatively smaller $t_{B}$ is found for the 2 and $17 \mathrm{~nm}$ wide rings. It indicates that the coupling between the electron-hole states in the TLM is largest in the $W=7 \mathrm{~nm}$ wide ring. Moreover, because $t_{B}$ in the GaAs/ $(\mathrm{Al}, \mathrm{Ga}) \mathrm{As}$ nanorings is larger, the excitonic $\mathrm{AB}$ oscillations are expected to be smaller than those in the (In,Ga)As/GaAs nanorings.

The dependence of $E_{x}^{-}$on $B$ extracted from the TLM is very close to the one obtained from the exact diagonalization approach, as the red solid and dashed curves in Fig. 7 show. This figure demonstrates that the excitonic $\mathrm{AB}$ oscillations decrease when $t$ increases. The excitonic AB oscillations are resolved according to the criterion that the second derivative of $E_{x}^{-}$with respect to $B$ changes sign at least once in the range $\left[0, B_{1}\right]$. That change arises from the anticrossing between the $1 S^{+}$and $2 S^{+}$states, but is present if the transfer integral does not exceed a critical value $t_{c r}$. The ranges of $t$ 

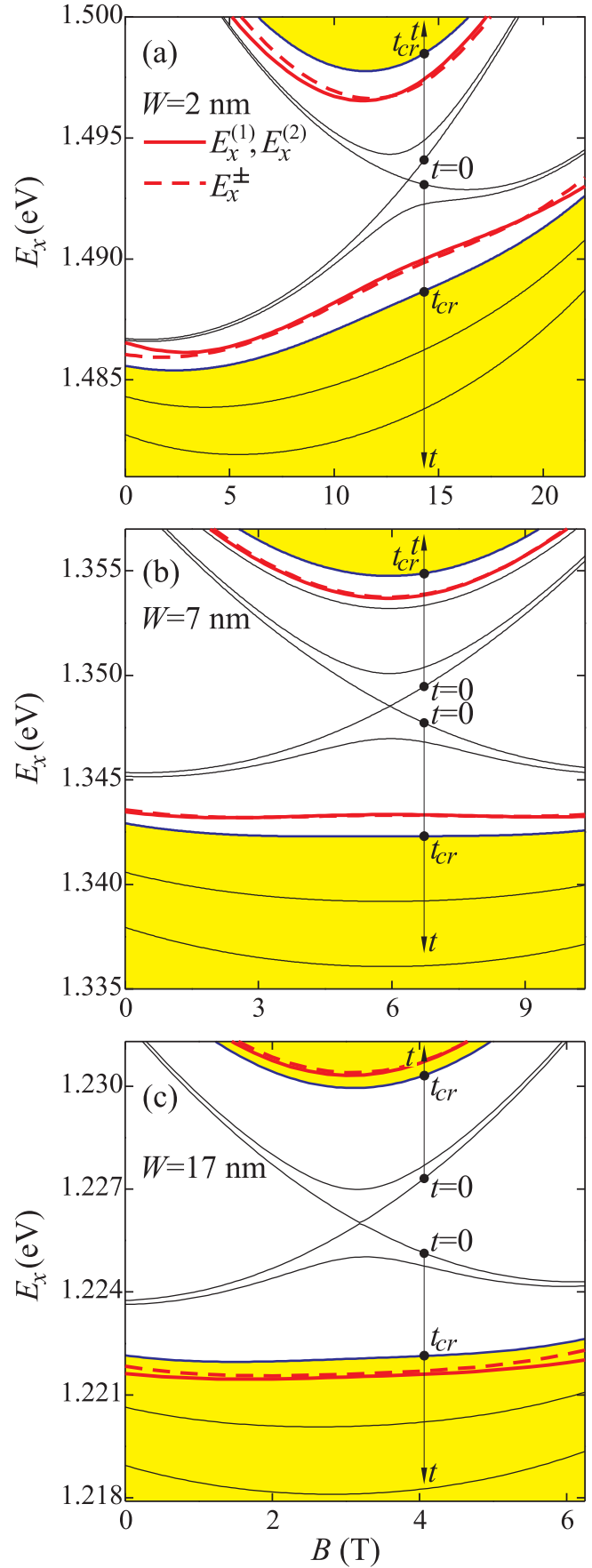

FIG. 7. (Color online) The variations of $E_{x}^{(1)}(B)$ and $E_{x}^{(2)}(B)$ functions with $t$ in (a) the $W=2 \mathrm{~nm}$, (b) the $W=7 \mathrm{~nm}$, and (c) the $W=17 \mathrm{~nm}$ wide (In,Ga)As/GaAs nanoring. No AB oscillations are present in the yellow regions, when $t \geqslant t_{c r}$. The solid red curves denote the exciton ground-state energies extracted from the exact diagonalization approach, whereas the dashed red curves are the TLM results.

where the excitonic $\mathrm{AB}$ oscillations do not occur are displayed as the yellow regions in Fig. 7. For all three cases in this figure the $E_{x}^{(1)}(B)$ curves are rather close to the critical case. Nevertheless, for the cases $W=2$ and $W=7 \mathrm{~nm}, t<t_{c r}$, and therefore oscillations in the exciton ground-state energy are found, whereas for $W=17 \mathrm{~nm} t>t_{c r}$, and the oscillations of $E_{x}^{(1)}$ as function of $B$ are absent in Fig. 7(c).
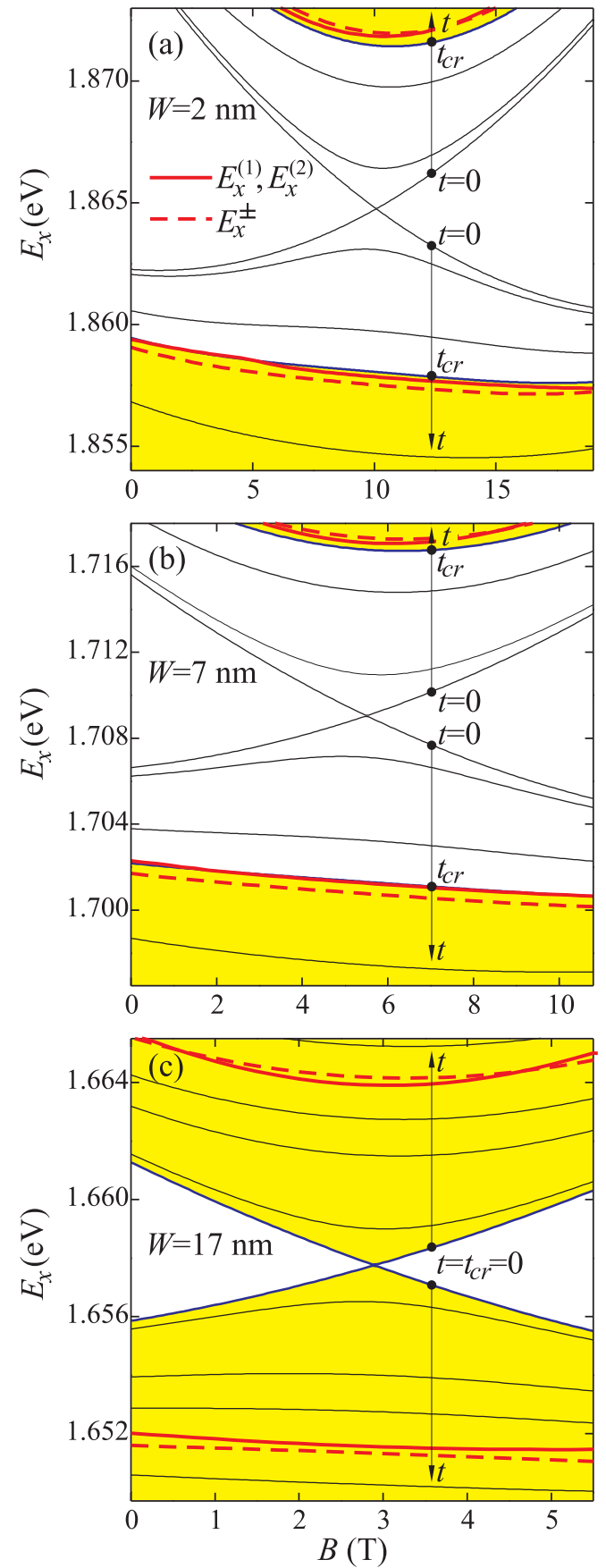

FIG. 8. (Color online) The same as Fig. 7, but now for the unstrained GaAs/(Al,Ga)As nanoring of width (a) $W=2 \mathrm{~nm}$, (b) $W=7 \mathrm{~nm}$, and (c) $W=17 \mathrm{~nm}$.

In $\mathrm{GaAs} /(\mathrm{Al}, \mathrm{Ga}) \mathrm{As}$ nanorings the exciton ground-state energy variation with $B$ is much smoother than in (In,Ga)As/GaAs nanorings, as is apparent from Fig. 8. Nevertheless, similar to Fig. 7 the results of the exact diagonalization calculations and the TLM are close to each other for all three values of the ring width. The TLM calculations reveal that excitonic $\mathrm{AB}$ oscillations do not appear for all three values of the ring width. However, the result for the $E_{x}^{(1)}$ variation with $B$ obtained with the exact diagonalization approach is very close to the $t_{c r}$ case for the $W=2$ and $7 \mathrm{~nm}$ wide rings, as shown in Figs. 8(a) and 8(b). On the other hand, no change of sign in the 

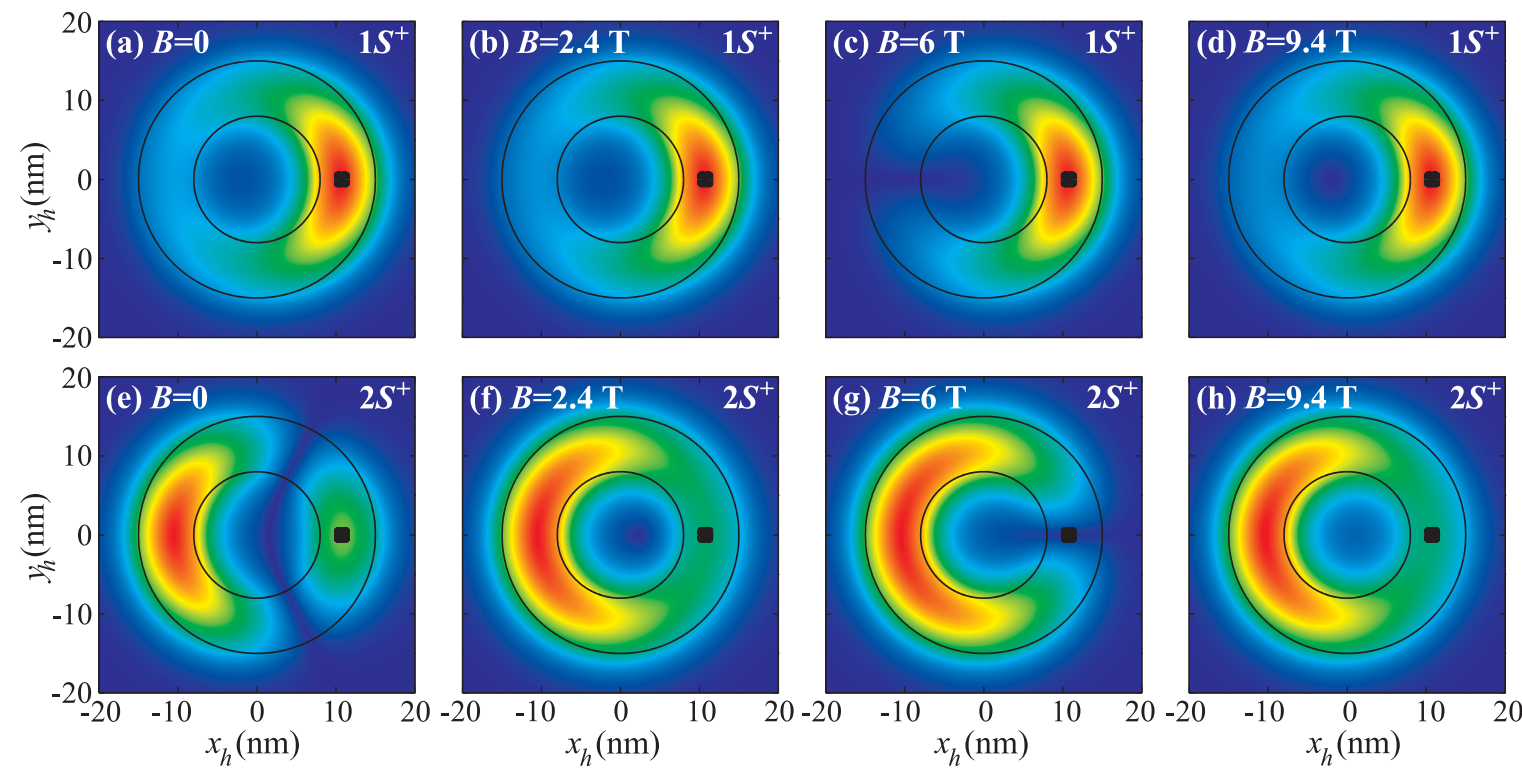

FIG. 9. (Color online) The CPD of the $1 S^{+}$and $2 S^{+}$exciton states in the plane of the $W=7 \mathrm{~nm}$ wide (In,Ga)As/GaAs nanoring for a few characteristic values of the magnetic field [see Fig. 3(b)]. The solid bullet denotes the position of the electron, and the two concentric circles denote the ring lateral boundaries. Top panels: The CPD of the $1 S^{+}$state for (a) $B=0$, (b) $B=2.4 \mathrm{~T}$, (c) $B=6 \mathrm{~T}$, and (d) $B=9.4 \mathrm{~T}$. Bottom panels: The CPD of the $2 S^{+}$state for (e) $B=0$, (f) $B=2.4 \mathrm{~T}$, (g) $B=6 \mathrm{~T}$, and (h) $B=9.4 \mathrm{~T}$.

quantity $d^{2} E_{x}^{-} / d B^{2}$ is found for $W=17 \mathrm{~nm}$, and thus $t_{c r}=0$, as Fig. 8(c) displays. Therefore, both employed models show that no $\mathrm{AB}$ oscillations are found in the exciton ground state for $\mathrm{GaAs} /(\mathrm{Al}, \mathrm{Ga}) \mathrm{As}$ nanorings. Furthermore, comparing Figs. 7 and 8 we find that excitonic $\mathrm{AB}$ oscillations exist only in a few $\mathrm{nm}$ wide strained rings.

Figure 9 shows the CPD of the $1 S^{+}$and $2 S^{+}$states in the $W=7 \mathrm{~nm}$ wide (In,Ga)As/GaAs nanoring for magnetic field values where the extrema of the $E_{x}^{(1)}$ dependence on $B$ are found in Fig. 3(b). The solid bullet indicates the fixed electron position, and the CPD is shown in the $\left(x_{h}, y_{h}\right)$ plane. One may notice that the hole in the $1 S^{+}$exciton state stays close to the electron [Figs. 9(a)-9(d)], whereas the two particles separate from each other in Figs. 9(e)-9(h). The CPD of the $2 S^{+}$state is characteristic of excited states, as previously found for the $2 \mathrm{D}$ rings. ${ }^{8}$ It indicates the possibility of the electron and hole tunneling through the Coulomb potential barrier in opposite directions. ${ }^{8}$ When the magnetic field increases, the CPD of both states changes. Yet, the CPD of the $2 S^{+}$ state exhibits larger variation with magnetic field, which is supported with the observed larger variation of the $E_{x}^{(2)}$ energy in the range $\left[0, B_{1}\right]$ in Fig. $3(\mathrm{~b})$. For $B=6 \mathrm{~T}$, where the $1 S^{+}$and $2 S^{+}$states anticross, the hole becomes maximally attracted to the electron in the $1 S^{+}$state, which is also demonstrated by the minimum of the $R_{\|}$dependence on $B$ in Fig. 4(c). One may also notice that because of the shape of the confining potential for the hole inside the ring opening [see Fig. 2(b)], the hole extends to the inner part of the ring as is also apparent from the CPD plots. The effective potential well for the hole allows its wave function to extends radially toward the ring center, whereas the electron is more localized in the ring. Therefore, the transfer integral is reduced due to different spatial localizations of the electron and the hole, which gives rise to oscillations of the exciton energy levels with $B$. Such a situation mimics type-II quantum dots, where the hole is localized inside the dot, and the Coulomb interaction confines the electron in the ring-shaped effective potential well around the barrier. The CPD variations with $B$ in the GaAs/(Al,Ga)As nanorings are similar to the ones displayed in Fig. 9.

\section{The oscillator strength and comparisons with experiment}

In addition to the exciton energy levels, the oscillator strength for the exciton recombination $f_{x}$ exhibits oscillations as a function of $B$, which is shown for the $1 S^{+}$state in Fig. 10(a). It has been already shown that increasing the magnetic field leads to a decrease of $R_{\|}$, and therefore $f_{x}$ increases. When $R_{\|}(B)$ has a minimum, $f_{x}(B)$ achieves a maximum. Variation of $f_{x}$ with $B$ shown in Fig. 10(a) seems to have the form $f_{x}=\bar{f}_{x}+\tilde{f}_{x}$, where $\bar{f}_{x}=a B+b$ is a linear function of $B$, and $\tilde{f}$ is the oscillatory residue. $\bar{f}_{x}(B)$ is displayed by the dashed line in Fig. 10(a), while $\tilde{f}_{x}=f_{x}-\bar{f}$ is shown in Fig. 10(b). The amplitude of oscillations is defined as the difference between the first maximum and the first minimum of $f_{x}$, which is denoted by $A_{f}$ in Fig. 10(b). Furthermore, $\Delta B$ in Fig. 5(b) denotes the interval of magnetic field between the first minimum and the first maximum [see Figs. 10(a) and 10(b)]. Both $A_{f}$ and $\Delta B$ are used to compute the relative amplitude of the first oscillation

$$
\delta f_{x}=A_{f} /\left\langle f_{x}\right\rangle,
$$

where $\left\langle f_{x}\right\rangle$ is the average value of $f_{x}$ in the interval $\Delta B$. Due to the population of higher exciton states, the oscillations of $I_{P L}$ are considerably smeared out, even at a temperature as low as $1 \mathrm{~K}$ [see Fig. 10(c)]. Such small oscillations might be very difficult to observe experimentally, but they resemble the $\mathrm{AB}$ oscillations of the photoluminescence intensity measured in type-II InP/GaAs quantum dots. ${ }^{15}$ 


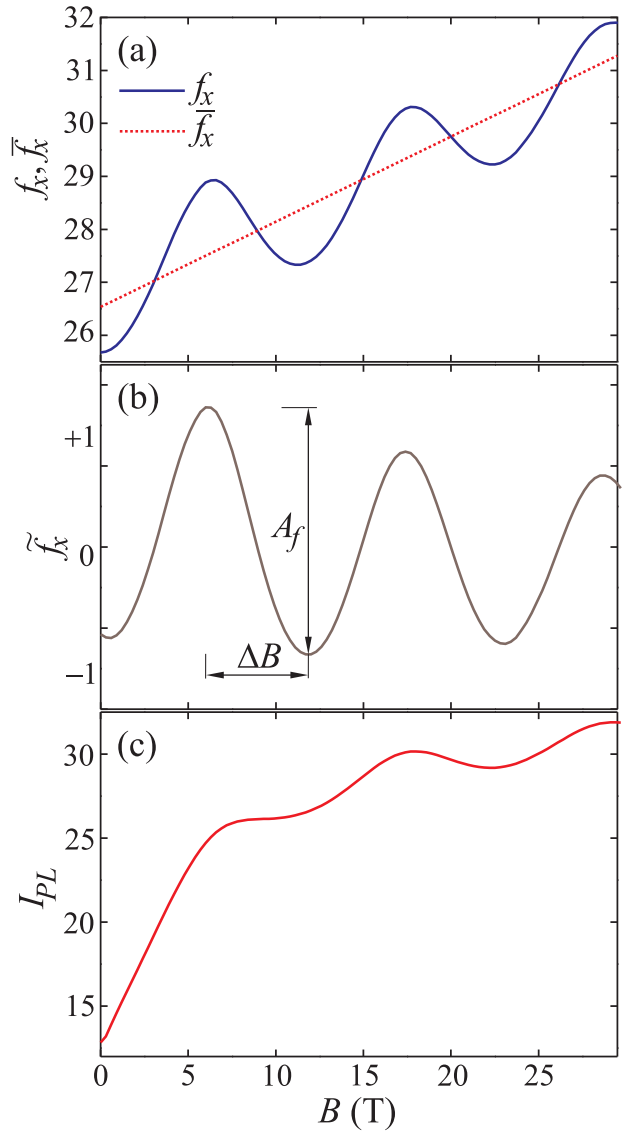

FIG. 10. (Color online) (a) The oscillator strength for recombination of the exciton ground state $f_{x}$ (solid curve) and the linear fitting curve $\bar{f}_{x}$ (dashed line) as function of magnetic field. (b) Variation of the residue $\tilde{f}_{x}=f_{x}-\bar{f}_{x}$ with magnetic field. (c) The photoluminescence intensity $I_{P L}$ exhibits weak oscillations as function of the magnetic field. The ring width equals $W=7 \mathrm{~nm}$ and the height is $h=4 \mathrm{~nm}$.

Figures 3(a)-3(c) illustrate that the magnitude of the $A B$ oscillations of the exciton ground-state energy depends on the width of the analyzed type-I ring. As a matter of fact, Fig. 11 shows that $\Delta_{x}$ and $\delta f_{x}$ of the exciton ground state are subject to changes when $W$ varies. The maximum of the $\Delta_{x}(W)$ curve equals $0.14 \mathrm{meV}$ and is located at $W=6 \mathrm{~nm}$, while $\delta f_{x}$ exhibits a maximum of $6.5 \%$ which is located at $W=5 \mathrm{~nm} . \delta f_{x}$ is a well-defined property of the exciton ground state in the whole explored range of $W$, from 2 to $22 \mathrm{~nm}$. On the other hand, $\Delta_{x}>0$ and $E_{x}^{(1)}$ vs $B$ dependence exhibits a second minimum only if the ring width is in the range from 4 to $10 \mathrm{~nm}$. Different domains of $\delta f_{x}$ and $\Delta_{x}$ imply that the optical excitonic $\mathrm{AB}$ effect could be present in type-I (In,Ga)As semiconductor nanorings, even though oscillations of the exciton ground-state level are not clearly visible. Previous analysis of concentric 1D rings showed that oscillations of the emission intensity for exciton recombination arise due to periodical bright-to-dark transitions of the exciton states; ${ }^{10}$ therefore $\delta f_{x}$ is much larger in concentric $1 \mathrm{D}$ rings. In the analyzed nanorings, the electron and the hole are localized in the same space; thus the exciton is only weakly polarized, and no bright-to-dark transitions are found. 3D nanorings,

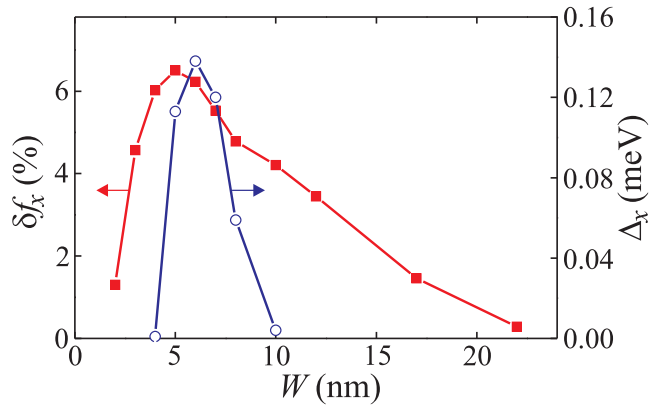

FIG. 11. (Color online) Variations of $\Delta_{x}$ (blue circles) and $\delta f_{x}$ (red squares) with the ring width.

therefore, offer different physics of the optical excitonic $A B$ effect than concentric $1 \mathrm{D}$ rings. ${ }^{10}$

There have been a few efforts to find excitonic AharonovBohm oscillations in the (In,Ga)As nanorings. The carefully prepared experiment of Ref. 16 failed to find this effect for neutral excitons, possibly because the ring width was large (of the order of $30 \mathrm{~nm}$ ). More recent work on excitons in $(\mathrm{In}, \mathrm{Ga}) \mathrm{As} / \mathrm{GaAs}$ nanorings produced a similar result, ${ }^{30}$ possibly due to large inhomogeneous broadening. The excitonic $\mathrm{AB}$ effect in $(\mathrm{In}, \mathrm{Ga}) \mathrm{As} / \mathrm{GaAs}$ nanorings was eventually discovered very recently. ${ }^{11,12}$ The amplitude of the exciton energy oscillations was a fraction of $\mathrm{meV}$, which is similar to what is found with our model. Furthermore, the shape of the observed experimental dependence of the exciton ground-state energy on $B$ [see Fig. 2(a) in Ref. 11] is similar to our Fig. 3(b). In order to explain the experimental data, an 1D ring model was used in Ref. 11. This model was able to produce only the regular oscillations of the exciton energy levels and the oscillator strength. However, the measured dependence of the photoluminescence intensity on magnetic field [see Fig. 2(c) in Ref. 11] showed a similar trend as in our Fig. 10. We note that the experiment presented in Ref. 11 is performed on an ensemble of (In, Ga)As/GaAs nanorings, whereas a single ring is considered in our theory. A qualitative similarity between our calculations and the experimental results of Ref. 11 indicate that the dimensions of the fabricated nanorings do not deviate much in the ensemble. The inhomogeneous broadening due to the size distribution is small enough that the separate peaks in the photoluminescence spectra arising from the two classes of nanorings of different size are resolved. The results of our theory compare favorably well with the measurements on rings with average radius $\left(R_{1}+R_{2}\right) / 2=11.6 \mathrm{~nm}$ in Ref. 11 which almost coincides with the average radius of the $W=7$ $\mathrm{nm}$ wide rings analyzed in the present work.

A good qualitative agreement between our results and measurements in Ref. 11 supports our previous conclusion that a 3D modeling is needed to compute the exciton energy levels and the oscillator strength for exciton recombination in type-I nanorings. For much closer quantitative comparison with experiment, the electron-hole exchange interaction should be included in the model. ${ }^{31}$ Previous measurements on the singly connected dots indicated that exciton energy level splitting could be of the order of $0.1 \mathrm{meV},{ }^{31}$ and is therefore comparable to the amplitude of the computed excitonic $\mathrm{AB}$ oscillations. Nevertheless, the exchange Hamiltonian does not depend on magnetic field ${ }^{31}$ and thus could not affect 
our findings about oscillations of the exciton states with magnetic field. More than electron-hole exchange interaction, exciton energy levels are affected by accurate measurements of dimensions and composition of nanorings. Interestingly enough, Ref. 11 demonstrates that, depending on the ring dimensions, oscillations of the photoluminescence intensity can be superimposed on a decaying background. Different behavior for two classes of nanorings was explained to arise from the possible ring elongation and the piezoelectric fields. ${ }^{11}$ These effects are, however, not included in our model, and taking them into account is beyond the scope of the present study. We also note that the experiment presented in Ref. 11 dealt with an ensemble of nanorings, while in Ref. 12 excitons in single type-I nanorings were investigated.

\section{CONCLUSION}

We showed theoretically that strain has a beneficial influence on the excitonic Aharonov-Bohm effect. The AharonovBohm oscillations of the exciton ground-state energy are found in an (In,Ga)As/GaAs nanoring, and they arise from anticrossings between the two lowest energy $L=0$ exciton states. The anticrossings can be explained by an approximate two-level Hamiltonian, which is derived by means of Löwdin perturbation theory. Existence of the oscillations is found to depend on the transfer integral due to the Coulomb interaction potential $t$. When $t$ is low, the energy gap between the two lowest energy $L=0$ exciton states becomes low, and the Aharonov-Bohm oscillations of the exciton ground-state energy become large. We found that strain is an important factor to establish these oscillations, by separating the hole from the electron, which establishes a condition similar to that found in type-II quantum dots. Furthermore, for nanorings of experimental inner radius and height, the amplitude of the exciton energy oscillations is found to depend on the ring width, which is maximum in a $6 \mathrm{~nm}$ wide nanoring, whereas no oscillations are found when the ring is wider than $10 \mathrm{~nm}$. The amplitude of the energy oscillations in the exciton ground state of an (In,Ga)As/GaAs nanoring could be of the order of $0.1 \mathrm{meV}$, whereas our calculations do not reveal the presence of exciton energy oscillations in unstrained $\mathrm{GaAs} /(\mathrm{Al}, \mathrm{Ga}) \mathrm{As}$ nanorings. Furthermore, the optical excitonic Aharonov-Bohm effect is present in (In,Ga)As/GaAs nanorings, but as oscillations in the oscillator strength for exciton recombination around a quasilinear dependence on the magnetic field. Such variations are qualitatively similar to what has been recently observed in Ref. 11. We infer that a proper design of type-I nanorings, e.g., varying their dimensions or by strain engineering, could lead to an enhancement of the excitonic Aharonov-Bohm effect.

\section{ACKNOWLEDGMENTS}

This work was supported by the Ministry of Education and Science of Serbia, the Flemish Science Foundation (FWO-Vl), the EU NoE: SANDiE, and the Belgian Science Policy (IAP). The calculations were performed on the CalcUA and Seastar computer clusters of the University of Antwerp. *milan.tadic@etf.bg.ac.rs

†nemanja.cukaric@etf.bg.ac.rs

${ }^{\ddagger}$ vladimir.arsoski@etf.bg.ac.rs

§rancois.peeters@ua.ac.be

${ }^{1}$ J. M. García, G. Medeiros-Ribeiro, K. Schmidt, T. Ngo, J. L. Feng, A. Lorke, J. Kotthaus, and P. M. Petroff, Appl. Phys. Lett. 71, 2014 (1997).

${ }^{2}$ A. Lorke, R. J. Luyken, A. O. Govorov, J. P. Kotthaus, J. M. García, and P. M. Petroff, Phys. Rev. Lett. 84, 2223 (2000).

${ }^{3}$ A. Chaplik, Pis'ma Zh. Éksp. Teor. Fiz. 62, 885 (1995) [JETP Lett. 62, 900 (1995)].

${ }^{4}$ R. A. Römer and M. E. Raikh, Phys. Rev. B 62, 7045 (2000).

${ }^{5}$ M. Grochol, F. Grosse, and R. Zimmermann, Phys. Rev. B 74, 115416 (2006).

${ }^{6}$ B. Li and F. M. Peeters, Phys. Rev. B 83, 115448 (2011).

${ }^{7}$ R. Okuyama, M. Eto, and H. Hyuga, Phys. Rev. B 83, 195311 (2011).

${ }^{8}$ H. Hu, J. L. Zhu, D. J. Li, and J. J. Xiong, Phys. Rev. B 63, 195307 (2001).

${ }^{9}$ J. Song and S. E. Ulloa, Phys. Rev. B 63, 125302 (2001).

${ }^{10}$ A. O. Govorov, S. E. Ulloa, K. Karrai, and R. J. Warburton, Phys. Rev. B 66, 081309 (2002).

${ }^{11}$ M. D. Teodoro, V. L. Campo Jr., V. Lopez-Richard, E. Marega Jr., G. E. Marques, Y. G. Gobato, F. Iikawa, M. J. S. P. Brasil, Z. Y.
AbuWaar, V. G. Dorogan, Yu. I. Mazur, M. Benamara, and G. J. Salamo, Phys. Rev. Lett. 104, 086401 (2010).

${ }^{12}$ F. Ding, N. Akopian, B. Li, U. Perinetti, A. Govorov, F. M. Peeters, C. C. Bof Bufon, C. Deneke, Y. H. Chen, A. Rastelli, O. G. Schmidt, and V. Zwiller, Phys. Rev. B 82, 075309 (2010).

${ }^{13}$ E. Ribeiro, A. O. Govorov, W. Carvalho Jr., and G. MedeirosRibeiro, Phys. Rev. Lett. 92, 126402 (2004).

${ }^{14}$ I. L. Kuskovsky, W. MacDonald, A. O. Govorov, L. Muroukh, X. Wei, M. C. Tamargo, M. Tadić, and F. M. Peeters, Phys. Rev. B 76, 035342 (2007).

${ }^{15}$ M. H. Degani, M. Z. Maialle, G. Medeiros-Ribeiro, and Evaldo Ribeiro, Phys. Rev. B 78, 075322 (2008).

${ }^{16}$ M. Bayer, M. Korkusinski, P. Hawrylak, T. Gutbrod, M. Michel, and A. Forchel, Phys. Rev. Lett. 90, 186801 (2003).

${ }^{17}$ N. Čukarić, M. Tadić, and F. M. Peeters, Superlattices Microstruct. 48, 491 (2010).

${ }^{18}$ N. A. J. M. Kleemans, I. M. A. Bominaar-Silkens, V. M. Fomin, V. N. Gladilin, D. Granados, A. G. Taboada, J. M. García, P. Offermans, U. Zeitler, P. C. M. Christianen, J. C. Maan, J. T. Devreese, and P. M. Koenraad, Phys. Rev. Lett. 99, 146808 (2007).

${ }^{19}$ S. Viefers, P. Koskinen, P. Singha Deo, and M. Manninen, Physica E 21, 1 (2004).

${ }^{20}$ J. H. Lee, Zh. M. Wang, Z. Y. Abuwaar, N. W. Strom, and G. J. Salamo, Nanotechnology 17, 3973 (2006). 
${ }^{21}$ M. Tadić, F. M. Peeters, K. L. Janssens, M. Korkusiński, and P. Hawrylak, J. Appl. Phys. 92, 5819 (2002).

${ }^{22}$ M. Tadić and F. M. Peeters, Phys. Rev. B 79, 153305 (2009).

${ }^{23}$ M. Tadić, F. M. Peeters, and K. L. Janssens, Phys. Rev. B 65, 165333 (2002); M. Tadić and F. M. Peeters, ibid. 70, 195302 (2004).

${ }^{24}$ I. Vurgaftman, J. R. Meyer, and L. R. Ram-Mohan, J. Appl. Phys. 89, 5815 (2001)

${ }^{25}$ J. H. Davies, The Physics of Low-dimensional Semiconductors (Cambridge University Press, 1997).

${ }^{26}$ J. I. Climente, J. Planelles, and W. Jaskólski, Phys. Rev. B 68, 075307 (2003).

${ }^{27}$ Al. L. Efros, M. Rosen, M. Kuno, M. Nirmal, D. J. Norris, and M. Bawendi, Phys. Rev. B 54, 4843 (1996).
${ }^{28}$ O. Stier, M. Grundmann, and D. Bimberg, Phys. Rev. B 59, 5688 (1999).

${ }^{29}$ M. Bayer, A. Kuther, A. Forchel, A. Gorbunov, V. B. Timofeev, F. Schäfer, J. P. Reithmaier, T. L. Reinecke, and S. N. Walck, Phys. Rev. Lett. 82, 1748 (1999).

${ }^{30}$ N. A. J. M. Kleemans, J. H. Blokland, A. G. Taboada, H. C. M. van Genuchten, M. Bozkurt, V. M. Fomin, V. N. Gladilin, D. Granados, J. M. García, P. C. M. Christianen, J. C. Maan, J. T. Devreese, and P. M. Koenraad, Phys. Rev. B 80, 155318 (2009).

${ }^{31}$ M. Bayer, G. Ortner, O. Stern, A. Kuther, A. A. Gorbunov, A. Forchel, P. Hawrylak, S. Fafard, K. Hinzer, T. L. Reinecke, S. N. Walck, J. P. Reithmaier, F. Klopf, and F. Schäfer, Phys. Rev. B 65, 195315 (2002). 